\title{
INFLUENCIA DA FARINHA DE MANGA NO CRESCIMENTO E COMPOSIÇÃO CORPORAL DA TILÁPIA DO NILO
}

\author{
INFLUENCE OF MANGO MEAL ON GROWTH AND BODY COMPOSITION \\ OF NILE TILAPIA
}

\author{
Souza, R.C. ${ }^{1}$; Melo, J.F.B. ${ }^{1 *}$; Nogueira Filho, R.M. ${ }^{2}$; Campeche, D.F.B. ${ }^{3}$ \\ e Figueiredo, R.A.C.R. ${ }^{4}$
}

\begin{abstract}
'Departamento de Zootecnia. Laboratório de Aquicultura. Universidade Federal do Vale do São Francisco (UNIVASF). Petrolina, PE. Brasil. *melojfb@yahoo.com.br

${ }^{2}$ Departamento de Engenharia de Pesca. Universidade do Estado da Bahia. Paulo Afonso, BA. Brasil. ${ }^{3}$ Empresa Brasileira de Pesquisa Agropecuária. EMBRAPA Semiárido. Petrolina, PE. Brasil. ${ }^{4} 3^{a}$ Superintendência Regional da CODEVASF. Petrolina, PE. Brasil.
\end{abstract}

\section{Palavras chave adicionais}

Alimento alternativo. Farinha de fruta. Nutrição de peixes. Oreochromis niloticus.

\section{RESUMO}

A farinha de manga com cascas foi avaliada como fonte de carboidrato em substituição ao milho para o crescimento de alevinos de tilápia e composição química da carcaça. Foram utilizados 180 peixes $(4,69 \pm 0,06 \mathrm{~g})$ estocados em 12 caixas de $500 \mathrm{~L}$, alimentados três vezes ao dia $(6 \%$ peso vivo), em um sistema de recirculação de água com biofiltro. Foram testadas quatro dietas com diferentes concentrações de farinha de manga $\mathrm{T} 1=$ dieta com $30 \%$ de milho; T2= $33 \%$ substituição milho por manga; T3= $66 \%$ substituição milho por manga e T4= $100 \%$ de substituição do milho pela manga com três repetições. Ao final de 45 dias, foram avaliados o desempenho zootécnico (peso médio final, ganho de peso médio final, taxa de crescimento específico, consumo de ração aparente, conversão alimentar aparente, rendimento de carcaça, sobrevivência) e composição química da carcaça. Os níveis de farinha de manga em substituição ao milho afetaram todas as variáveis de desempenho a partir de $33 \%$ de substituição $(p<0,05)$, com exceção da sobrevivência e o rendimento de carcaça $(p>0,05)$. Os valores da composição química da carcaça foram alterados com exceção da matéria mineral. A farinha de manga em substituição ao milho pode ser utilizada em até $33 \%$ na ração da tilápia do Nilo sem prejudicar o desempenho zootécnico e a composição química da carcaça.

Recibido: 12-4-12. Aceptado: 12-9-12.

\section{Additional KEYWORDS}

Alternative food. Fish nutrition. Fruit meal. Oreochromis niloticus.

\section{SUMMARY}

Mango meal with peel was evaluated as carbohydrate source in substitution of corn, for tilapia juvenile growth and carcass composition. There were used 180 fish $(4,69 \pm 0,06 \mathrm{~g})$ in 12 tanks of $500 \mathrm{~L}$, fed three times a day $(6 \%$ of live weight), in a water recirculating system with biofilter. Four diets were evaluated with different mango meal concentrations $\mathrm{T} 1=$ diet with $30 \%$ of corn; and T2, T3 and T4 in which 33, 66, and $100 \%$ of corn was replaced by mango meal. All of the treatments had three repetitions. At the end of 45 days performance was evaluated (final weight, weight gain; specific growth rate, feed consumption, apparent feed conversion rate, carcass yield, survival) and chemical carcass composition. The levels of mango meal substitution by corn meal affected all the variables analyzed from level $33 \%(p<0,05)$, with the exception of survival and carcass yield ( $>>0,05)$. Carcass chemical composition values were changed except for ash. Mango meal in substitution for corn may be used until the level of $33 \%$ in Nile tilapia feed without damage for growth performance and carcass chemical composition.

\section{INTRODUÇÃO}

O alto custo das rações é um dos fatores limitantes na piscicultura. Sua redução é 
primordial para o sucesso comercial da atividade. Uma opção para substituir as fontes proteicas animais com fins energéticos na dieta dos peixes são os ingredientes de origem vegetal por serem ricas em carboidratos (Enes et al., 2011). Os carboidratos são importantes fontes energéticas quando comparado às proteínas pelo seu baixo custo (Liti et al., 2006), entretanto, os peixes possuem capacidade limitada na utilização deste nutriente, que depende do tipo e hábito alimentar do animal, porém quando utilizados em níveis adequados, pode poupar o uso da proteína para o crescimento (Honorato et al., 2009).

O milho, fonte tradicional de carboidratos na alimentação de peixes, contém entre 70 e $80 \%$ de amido (Rostagno et al., 2005). Fontes alternativas podem ser utilizadas como a manga em forma de farinha. Segundo Bernardes-Silva et al. (2003) em alguns cultivares de manga, a frutose é o monossacarídeo mais abundante e pode variar entre 2,3 a $3,1 \%$.

A manga é cultivada em várias regiões do Brasil, principalmente na região Nordeste, por apresentar excelentes condições para o seu desenvolvimento e produção (Coelho et al., 2002) destacando-se a região do Vale do São Francisco. Esta região apresenta ampla disponibilidade dessa fruta, com grande volume de exportação, no entanto as perdas no processo de produção e comercialização chegam entre 20 e $40 \%$ (Choudhury, 1995; Perosa et al., 2009) e muitas vezes são descartadas no ambiente sem prévio tratamento causando prejuízos econômicos significativos além de problemas ambientais. Entretanto estes frutos impróprios ao consumo humano possuem potencial nutricional e podem ser aproveitados e utilizados como fonte de carboidratos na elaboração de rações para peixes. De acordo com Monteiro (2009) a manga contém 10,97 e 14,36 g/100 g de carboidratos; 11,71 e $22,5 \mathrm{mg} / 100 \mathrm{~g}$ de vitamina $\mathrm{C}$, e energia de 50,02 e 58,12 kcal em g/100 g na polpa e casca respectivamente.
A utilização de alguns ingredientes regionais nas rações para peixes tais como: coco (Santos et al., 2009), farelo do resíduo de manga (Lima et al., 2011), folhas de mandioca, vagem de algaroba (Jesus et al., 2011), resíduo de café (Pimenta et al., 2011) dentre outros já vem sendo estudadas, contudo estas pesquisas devem ser intensificadas principalmente visando reduzir custos na alimentação dos peixes.

A avaliação de ingredientes não convencionais é importante devido à possibilidade de se propor tecnologias apropriadas às necessidades econômicas de cada região. Estes ingredientes não devem competir com a alimentação humana e que seja preferencialmente subproduto da mesma (Teixeira et al., 2006). Agregar valor aos resíduos, subprodutos ou coprodutos provenientes seja da indústria processadora de frutas, exportações, pós-colheitas, rejeitos de feiras livres ou supermercados podem representar ganhos econômicos, ambientais, tanto para produtores como piscicultores. Diante deste contexto, objetivou-se nesta pesquisa avaliar a utilização da farinha de manga com cascas em substituição à farinha de milho no desempenho e composição corporal de alevinos de tilápia do Nilo.

\section{MATERIALE MÉTODOS}

O experimento foi executado nas instalações do Laboratório de Aquicultura no Campus de Ciências Agrárias da UNIVASF durante 45 dias. Foram utilizados 180 juvenis de tilápia do Nilo com peso inicial de 4,69 \pm $0,06 \mathrm{~g}$ oriundos do Centro Integrado de recursos Pesqueiros e Aquicultura de Bebedouro, $3^{\mathrm{a}}$ Superintendência regional da Companhia de Desenvolvimento dos Vales do São Francisco e do Parnaíba. Os peixes foram distribuídos em 12 caixas d'água circular de polietileno $(500 \mathrm{~L})$. Estas caixas estavam acopladas a um sistema de recirculação de água com uso de filtro mecânico e biológico.

Os animais passaram por um período de 


\section{FARINHA DE MANGA E CRESCIMENTO E COMPOSIÇÃO CORPORAL DA TILÁPIA}

adaptação de cinco dias, alimentados com ração comercial contendo $32 \%$ de proteína bruta. Os tratamentos consistiram de níveis crescentes de farinha de manga com cascas em substituição ao milho (tabela I). Para a elaboração da farinha de manga, foram utilizadas mangas maduras da variedade Tommy Atkins. As frutas foram lavadas, cortadas em pedaços pequenos e retirado o caroço. Após colocou-se em estufa de ventilação forçada a $55^{\circ}$ por 86 horas. Posteriormente foi realizada a moagem em moinho tipo facas em peneiras de $1 \mathrm{~mm}$. Para a elaboração das rações, os ingredientes secos foram previamente misturados, adicionado óleo de soja, umedecidos com aproximadamente $20 \%$ de água e posterior peletização. Após a peletização, as rações foram levadas à estufa de ventilação forçada a $55^{\circ} \mathrm{C}$ por um período de 36 horas, e depois mantidas refrigeradas. Para o fornecimento aos animais os peletes foram desintegrados em moinho manual, passados em peneiras de diversas malhas para obtenção de granulometrias adequadas ao diâmetro da boca dos peixes.

Diariamente foram monitorados a temperatura da água, o $\mathrm{pH}$ e a condutividade elétrica por meio de aparelhos portáteis. A temperatura da água foi de 26,36 $\pm 0,71 \mathrm{e}$ $27,63 \pm 0,53^{\circ} \mathrm{C} ; \mathrm{o} \mathrm{pH}$ situou-se entre $7,28 \pm$ 0,52 e $7,20 \pm 0,51$; e a condutividade elétrica

Tabela I. Composição (\%) das dietas experimentais. (Composition (\%) of experimental diets).

\begin{tabular}{|c|c|c|c|c|}
\hline & \multicolumn{4}{|c|}{ Nível de substituição do milho por manga (\%) } \\
\hline & 0 & 33 & 66 & 100 \\
\hline Farelo de soja & 61,68 & 61,68 & 61,68 & 61,68 \\
\hline Milho moído & 30,00 & 20,00 & 10,00 & 0,00 \\
\hline Farinha de manga & 0,00 & 10,00 & 20,00 & 30,00 \\
\hline Fosfato bicálcico & 3,03 & 3,03 & 3,03 & 3,03 \\
\hline Metionina & 0,50 & 0,50 & 0,50 & 0,50 \\
\hline Óleo de soja & 2,23 & 2,23 & 2,23 & 2,23 \\
\hline Premix vitamínico e mineral ${ }^{1}$ & 2,00 & 2,00 & 2,00 & 2,00 \\
\hline Sal & 0,50 & 0,50 & 0,50 & 0,50 \\
\hline Vitamina $\mathrm{C}^{2}$ & 0,05 & 0,05 & 0,05 & 0,05 \\
\hline BHT (Butil hidroxi tolueno) & 0,01 & 0,01 & 0,01 & 0,01 \\
\hline Total & 100,00 & 100,00 & 100,00 & 100,00 \\
\hline \multicolumn{5}{|c|}{ Composição calculada das dietas } \\
\hline Energia digestível kcal/kg & 2,717 & 2,913 & 3,110 & 3,307 \\
\hline Proteína bruta* & 34,30 & 33,03 & 32,38 & 31,11 \\
\hline Cálcio & 0,98 & 0,97 & 0.97 & 0.97 \\
\hline P (fósforo disponível) & 0,69 & 0,68 & 0,67 & 0,67 \\
\hline Lisina total & 1,84 & 1,82 & 1,79 & 1,77 \\
\hline Metionina total & 0,96 & 0,94 & 0,92 & 0,90 \\
\hline
\end{tabular}

${ }^{1}$ Premix vitamínico e mineral (Supremais, Campinas-SP): Composição por quilo: vit. A=1200 000 UI; vit. D3= $200000 \mathrm{UI}$; vit. E= 12000 mg; vit. K3= $2400 \mathrm{mg}$; vit. $B 1=4800 \mathrm{mg}$; vit. $B 2=4800 \mathrm{mg}$; vit. $B 6=4000$ $\mathrm{mg}$; vit. $\mathrm{B} 12=4800 \mu \mathrm{g}$; ác. fólico $=1200 \mathrm{mg}$; pantotenato de cálcio $=12000 \mathrm{mg}$; vit. $\mathrm{C}=48000 \mathrm{mg}$; biotina $=$ $48 \mathrm{mg}$; colina $=65000 \mathrm{mg}$; ácido nicotínico= $24000 \mathrm{mg} ; \mathrm{Fe}=10000 \mathrm{mg} ; \mathrm{Cu}=600 \mathrm{mg} ; \mathrm{Mn}=4000 \mathrm{mg} ; \mathrm{Zn}=$ $6000 \mathrm{mg}$; I= $20 \mathrm{mg} ; \mathrm{Co}=2 \mathrm{mg}$ e Se= $20 \mathrm{mg}$.

${ }^{2}$ Vitamina C (Basf, São Paulo-SP): sal cálcica 2-monofosfato de ácido ascórbico, 42 \% de princípio ativo; *Valores analisados (Silva e Queiroz, 2002). 
foi de $123 \pm 6,1$ e $122 \pm 6,5 \mu \mathrm{S} / \mathrm{cm}$ nos períodos manhã e tarde respectivamente. As caixas d'água foram sifonadas pela manhã e à tarde antes do arraçoamento, com troca de aproximadamente $10 \%$ do total da água com o objetivo de remoção de fezes e sobras de ração. $\mathrm{O}$ fornecimento da ração foi na proporção de $6 \%$ da biomassa, distribuídos três vezes ao dia. A partir da taxa de arraçoamento ofertada, calculou-se a quantidade de ração fornecida aos peixes e estimou-se o consumo aparente de ração. A avaliação da conversão alimentar aparente foi realizada através da relação entre a quantidade de ração ofertada e o ganho de peso ao longo do período experimental. A cada 15 dias foram realizadas biometrias para acompanhamento do crescimento dos animais e ajuste da ração a ser ofertada.

Ao final do período experimental todos os animais foram pesados para coleta de dados de crescimento, com posterior insensibilização com água e gelo, e foram sacrificados por secção medular. A análise da composição corporal dos peixes foi realizada pela desidratação das carcaças em estufa de ventilação forçada $\left(55^{\circ} \mathrm{C}\right)$ por 72 horas e trituradas. Foram determinados a matéria seca, proteína bruta, extrato etéreo e cinzas de acordo com técnica descrita pela AOAC (1990). As variáveis zootécnicas avaliadas foram: peso médio final, ganho peso médio total, consumo total de ração aparente, conversão alimentar aparente, taxa de crescimento específico, sobrevivência, rendimento de carcaça, e a composição química da carcaça dos peixes.

Os dados obtidos foram submetidos à análise de variância com nível de $5 \%$ significância. Quando verificado diferença significativa procedeu-se à análise de regressão. Utilizou-se o programa assistência estatística ASSISTAT 7.5 beta (Silva, 2010).

\section{RESULTADOSEDISCUSSÃO}

Os valores médios do desempenho zootécnicos estão descritos na (tabela II).
Todas as variáveis analisadas com exceção da sobrevivência e do rendimento de carcaça apresentaram diferenças significativas $(\mathrm{p}<0,05)$.

Foi observado menor peso final nos animais que se alimentaram com a ração contendo 66 e $100 \%$ da farinha de manga como fonte de carboidrato. Concentrações baixas da farinha de manga com cascas, em substituição ao milho foram mais eficientes no desempenho dos peixes. Resposta semelhante foi obtida por Lima et al. (2009) ao utilizarem farelo de vagem de algaroba substituindo parcialmente o milho na dieta de tilápias, observaram redução significativa no ganho de peso e consumo de ração à medida que aumentou os níveis do farelo, entretanto não observaram efeito na conversão alimentar aparente. Lima et al. (2011) incluíram níveis de 0,5, 10 e $15 \%$ de farelo de resíduo de manga na ração de tilápia e não observaram diferença significativa no ganho de peso. $\mathrm{O}$ mesmo fato ocorreu com níveis de 10 e $20 \%$ de farelos da vagem de algaroba e folha da mandioca para tilápias, os quais não alteraram o desempenho (Jesus et al., 2011). Melo et al. (2012), avaliaram a inclusão de farinha de manga sem cascas substituindo o milho, concluíram que a substituição de $100 \%$ do milho por manga não prejudica o desempenho. $\mathrm{O}$ farelo de resíduo de manga uma fonte alternativa, possui baixos teores de lipídios, minerais e proteínas (Vieira et al., 2008), entretanto possui valores energéticos de $3724 \mathrm{kcal} / \mathrm{kg}$ de ração (Lima et al., 2011) bem próxima à energia contida no milho (3886 kcal $/ \mathrm{kg}$ de ração) em rações para peixes (Oliveira Filho e Fracalossi, 2006). Como pode ser observado na (tabela I), as rações com maiores concentrações de energia foram as que continham farinha de manga. É provável que a fonte de carboidrato testada neste experimento tenha prejudicado o desempenho da tilápia pela sua composição em fibra ou algum fator antinutricional contido na mesma. Monteiro (2009) avaliou o valor nutricional de partes 


\section{FARINHA DE MANGA E CRESCIMENTO E COMPOSIÇÃO CORPORAL DA TILÁPIA}

convencionais e não convencionais de frutas e hortaliças, observou teores de fibra de 4,0 e $12,24 \%$ na polpa e casca respectivamente na manga Tommy Atkins. Segundo Pimenta et al. (2011) a utilização de resíduos na alimentação animal pode ser um problema devido a altas quantidades de fibra e fatores antinutricionais, o que pode prejudicar o desempenho dos animais. Altos teores de fenólicos totais foram encontrados no farelo do resíduo de manga (Vieira et al., 2008). Em altas concentrações, estes compostos podem reduzir o valor nutricional do alimento, além de inibir as enzimas digestivas (Shahidi e Naczk, 1995; Getachew et al., 2000), o que pode limitar a substituição total do milho pela manga com a casca. Lima et al. (2011), relataram que a fibra do farelo de resíduo de manga pode ter influenciado a digestibilidade deste alimento para a tilápia.
Além da composição da fibra no alimento, o tipo de carboidrato também pode influenciar o desempenho. Muñoz-Ramírez (2005) avaliou vários tipos de carboidratos na alimentação do pacu, e verificou que o melhor desempenho foi com $40 \%$ de fécula pré-gelatinizada e modificada. A composição do amido em amilose e amilopectina no carboidrato pode influenciar diretamente o desempenho (Wilson, 1994). No estudo de Rawles e Lochmann (2003) a relação amilopectina/amilose de $30 \% / 70 \%$ promoveu maior peso em sunshine bass (Morone chrysops x $M$. saxatilis). Desta forma, a farinha de manga certamente apresenta uma composição diferenciada em termos de carboidratos e isto pode ter influenciado o desempenho.

A taxa de crescimento específico apresentou diferença significativa $(\mathrm{p}<0,05)$

Tabela II. Valores médios do desempenho e das características de carcaça de alevinos de tilápia do Nilo alimentadas com diferentes níveis de inclusão de farinha de manga em substituição ao milho. (Mean values of performance and carcass characteristics of Nile tilapia fingerlings fed different levels of inclusion of mango meal substitution of corn).

\begin{tabular}{|c|c|c|c|c|c|c|}
\hline \multicolumn{7}{|c|}{ Nível de substituição do milho por manga (\%) } \\
\hline & 0 & 33 & 66 & 100 & Equação de regressão & $\mathrm{R}^{2}$ \\
\hline PMI $(\mathrm{g})^{*}$ & $4,66 \pm 0,13$ & $4,67 \pm 0,09$ & $4,72 \pm 0,08$ & $4,74 \pm 0,12$ & & \\
\hline $\operatorname{PMF}(\mathrm{g})$ & $37,51 \pm 0,75^{a}$ & $35,84 \pm 2,11^{\mathrm{a}}$ & $32,04 \pm 1,34^{b}$ & $22,58 \pm 0,96^{c}$ & $Y=-4,376 x+42,53$ & 0,81 \\
\hline GPMF (g) & $32,85 \pm 0,88^{a}$ & $31,17 \pm 2,07^{a}$ & $27,32 \pm 1,34^{b}$ & $17,84 \pm 0,84^{c}$ & $Y=-4,885 x+39,51$ & 0,88 \\
\hline TCE (\%) dia & $4,63 \pm 0,11^{\mathrm{a}}$ & $4,52 \pm 0,12^{\mathrm{a}}$ & $4,25 \pm 0,10^{\mathrm{b}}$ & $3,46 \pm 0,04^{c}$ & $Y=-0$, & 0,85 \\
\hline CTRA (g) & $33,92 \pm 1,42^{\mathrm{a}}$ & $33,34 \pm 0,94^{\mathrm{a}}$ & $32,46 \pm 1,38^{\mathrm{a}}$ & $26,95 \pm 1,33^{b}$ & 7,115 & 0,77 \\
\hline CAA & $1,03 \pm 0,03^{c}$ & $1,07 \pm 0,05^{c}$ & $1,19 \pm 0,01^{b}$ & $1,51 \pm 0,02^{a}$ & $Y=0,156 x+0,81$ & 0,85 \\
\hline $\mathrm{RC}(\%)^{*}$ & $85,29 \pm 1,68$ & $85,36 \pm 1,7$ & $86,57 \pm 1,74$ & $84,87 \pm 1,98$ & & \\
\hline $\mathrm{S}(\%)^{*}$ & $97,78 \pm 3,85$ & $97,78 \pm 3,8$ & $97,78 \pm 3,85$ & $100 \pm 0,00$ & & \\
\hline \multicolumn{7}{|c|}{ Composição química carcaça (\% matéria natural) } \\
\hline PB & $59,50 \pm 1,24^{a}$ & $59,94 \pm 0,62^{a}$ & $61,69 \pm 0,62^{a}$ & $54,36 \pm 0,16^{\mathrm{b}}$ & $Y=-1,943 x^{2}+8$ & 0,81 \\
\hline EE & $20,42 \pm 0,08^{\mathrm{ab}}$ & $21,49 \pm 0,24^{a}$ & $19,71 \pm 0,53^{b}$ & $19,27 \pm 0,08^{b}$ & $Y=-0,378 x^{2}+1,365 x+19,643$ & 0,68 \\
\hline $\mathrm{MM}^{*}$ & $15,46 \pm 0,05$ & $14,62 \pm 0,08$ & $15,65 \pm 0,08$ & $17,06 \pm 1,28$ & & \\
\hline
\end{tabular}

$\mathrm{PMI}=$ peso médio inicial; $\mathrm{PMF}=$ peso médio final; $\mathrm{GPMT}=$ gaho de peso médio total; $\mathrm{TCE}=$ taxa de crescimento específico; CTRA = consumo total ração aparente; $C A A=$ conversão alimentar aparente; $\mathrm{RC}=$ rendimento de carcaça; $\mathrm{S}=$ sobrevivência. $\mathrm{PB}=$ proteína bruta; $\mathrm{EE}=$ extrato etéreo; $\mathrm{MM}=$ matéria mineral.

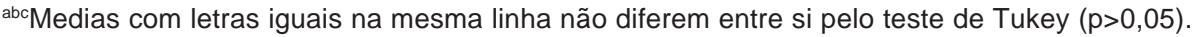


entre os tratamentos. A menor taxa de crescimento específico nos peixes foi com a dieta contendo 66 e $100 \%$ de farinha de manga em substituição ao milho. Lemos et al. (2011) incluíram farelo de coco em dietas para o tambaqui obtiveram menores valores entre 1,85 a 1,99 contra 3,46 a 4,63 neste trabalho. Oishi (2007) também obteve valores inferiores que variaram entre 1,9 a 2,2 em tambaquis (Colossoma macropomum) alimentados com farinha de resíduo de castanha da Amazônia.

Quanto ao consumo total de ração aparente, observou-se que nos níveis 0,33 e $66 \%$ de substituição do milho por farelo de manga não apresentaram diferença significativa $(p>0,05)$. Entretanto, foi menor no tratamento composto por $100 \%$ de manga $(\mathrm{p}<0,05)$. Nos estudos de outras fontes de carboidratos por Ramos et al. (2008), o aumento da inclusão de farinha da parte aérea da mandioca na alimentação de tilápias, reduziu o consumo de forma linear. Lima et al. (2011) alimentaram tilápia com diferentes níveis de farelo de resíduo de manga e não observaram alterações no consumo de ração, diferindo portanto do presente trabalho. É provável que a redução do consumo pelo aumento da farinha de manga neste trabalho até $66 \%$ tenha sido influenciada pela composição do carboidrato da manga, à palatabilidade das dietas ou algum fator antinutricional. De acordo com Vieira et al. (2008) os compostos fenólicos estão presentes no farelo do resíduo de manga. Estes compostos são responsáveis pelo sabor amargo do alimento (Shahidi e Naczk, 1995). Este fato pode ter influenciado palatabilidade causando redução no consumo da ração.

A conversão alimentar reflete a eficiência de um alimento ou dieta. Os valores de conversão alimentar obtidos foram: 1,03; 1,$07 ; 1,19$ e 1,51 . Esta sofreu influencia dos níveis da farinha de manga em substituição ao milho ( $\mathrm{p}<0,05)$. Os melhores valores foram obtidos nas concentrações de 0 e $33 \%$ (tabela II). Nas concentrações de 66 e $100 \%$ a conversão alimentar foi menos eficiente. Tachibana et al. (2010) não observaram diferenças ao avaliarem a conversão alimentar de tilápias alimentadas com triticale em substituição ao milho. Lima et al. (2011) não encontraram diferença significativa na conversão alimentar de tilápias alimentadas com resíduo de manga, embora os valores tenham sido superiores ao deste estudo. Gonçalves et al. (2002) obtiveram respostas semelhantes com piores valores de conversão alimentar aparente à medida que aumentou os níveis de farelo de canola na dieta de piavuçu (Leporinus macrocephalus).

Os piores resultados na conversão alimentar nos tratamentos 66 e $100 \%$ pode estar relacionado aos níveis de fibra na ração, a composição ou a palatabilidade. De acordo com Pereira-da-Silva e Pezzato (2000) ao avaliarem o grau de atrato-palatabilidade de vários alimentos para tilápia afirmaram que esta deve ser realizada considerandose uma combinação de parâmetros em relação ao comportamento alimentar. Desta forma, o paladar pode ser considerado o fator responsável pela seleção final, definindo a ingestão ou a rejeição de um alimento, bem como, a quantidade a ser consumida (Adams etal., 1988).

O rendimento de carcaça não sofreu influência $(p>0,05)$ dos níveis de farinha de manga em substituição ao milho. Resultado semelhante foi obtido por Lima et al. (2011) ao ofertarem farelo do resíduo de manga para a tilápia do Nilo. Também foi verificado por Pedron et al. (2011), que o rendimento de carcaça de jundiá não é influenciado pelas diferentes proporções de carboidratos amilose:amilopectina.

A sobrevivência não foi afetada pelos níveis de manga substituindo o milho na ração $(p>0,05)$. Estes resultados corroboram os estudos de Azaza et al. (2008), que não observaram diferença significativa na taxa de sobrevivência dos animais alimentados com níveis crescentes de farinha de tâmara em contraponto a níveis decrescentes de soja na ração da tilápia. 


\section{FARINHA DE MANGA E CRESCIMENTO E COMPOSIÇÃO CORPORAL DA TILÁPIA}

Os valores da composição química da carcaça foram alterados $(p<0,05)$ com exceção da matéria mineral (tabela II). A proteína bruta e o extrato etéreo foram menores nos animais que receberam a ração contendo $100 \%$ de farinha de manga. Signor et al. (2007) não observaram diferenças significativas para a proteína bruta, extrato etéreo e matéria mineral ao utilizarem farinha de vísceras de aves na alimentação do piavuçu. Na literatura pouco é descrito sobre os tipos de carboidratos e a composição química da carne do peixe, pois a maior parte esta relacionada à energia, proteína e lipídio na ração. Neste estudo, as concentrações de carboidratos testadas na ração modificaram o perfil químico da carne. Em trabalho realizado por Austreng et al. (1997) os níveis de carboidratos na dieta para truta diminuíram gordura e energia, e aumentaram proteína e cinzas. Fabregat et al. (2011) ao avaliarem a substituição de farinha de peixe por farelo de soja na dieta observaram redução nos teores de proteína bruta da carcaça em juvenis de curimba (Prochilodus lineatus).

A composição química da carcaça é refletida pelos nutrientes contidos na dieta,

\section{BIBLIOGRAFIA}

Adams, M.A.; Johnsen, P.B. and Hong-QI, Z. 1988. Chemical enhancement of feeding for the herbivorous fish Tilapia zillii. Aquaculture, 72: 95-107.

AOAC. 1990. Official methods of analysis. $15^{\mathrm{a}} \mathrm{ed}$ Association of Official Analytical Chemists. Washington. $1018 \mathrm{pp}$.

Austreng, E.; Risa, S.; Edwards, D.J. and Hvidsten, H. 1977. Carbohydrate in rainbow trout diets. II. Influence of carbohydrate levels on chemical composition and feed utilization of fish from different families. Aquaculture, 11: 39-50.

Azaza, M.S.; Mensi, F.; Kammoun, W.; Abdelouaheb, A.; Brini, B. and Kraïem, M. 2008. Nutritional evaluation of waste date fruit as partial substitute for soybean meal in practical diets of juvenile Nile tilapia, Oreochromis logo o desbalanceamento dos mesmos pode influenciá-la (Signor et al., 2007). A ração contendo $100 \%$ de farinha de manga provavelmente não esteve nutricionalmente balanceada o que diminuiu a síntese protéica e o extrato etéreo na carcaça dos animais.

\section{CONCLUSÕES}

Até $33 \%$ de farinha de manga com cascas pode ser adicionado à ração sem comprometer o desempenho zootécnico e a composição química da carcaça para tilápia do Nilo, apresentando-se como potencial fonte alternativa energética na dieta para a espécie. Estudos de digestibilidade, bem como a investigação de fatores antinutricionais são necessários para garantir o uso deste ingrediente pela indústria de rações.

\section{AGRADECIMENTOS}

À Fundação de Amparo à Ciência e Tecnologia do Estado de Pernambuco (FACEPE) pela bolsa de mestrado concedida ao $1^{\circ}$ autor. Ao Centro Integrado de recursos Pesqueiros e Aquicultura de Bebedouro $-3^{\mathrm{a}}$ Superintendência regional da CODEVASF-PE pela doação dos alevinos.

niloticus L. Aquac Nutr, 15: 262-272.

Bernardes-Silva, A.P.F.; Lajolo, F.M. e Cordenunsi, B.R. 2003. Evolução dos teores de amido e açúcares solúveis durante o desenvolvimento e amadurecimento de diferentes cultivares de manga. Ciênc Tecnol Aliment, 23 (supl): 116120.

Coelho, E.P.; Oliveira, F.C.; Nascimento, C.J.A.; Vasconcelos, L.F.L. e Araújo, E.C.E. 2002. A cultura da manga sob diferentes regimes de profundidades do lençol freático em condições subúmidas. Rev Bras Frutic, 24: 96-100.

Choudhury, M.M. 1995. Perdas de frutas e hortaliças na pós-colheita. Informe CPATSA, Petrolina, PE. 2 (20): 2.

Enes, P.; Panserat, S.; Kaushik, S. and OlivaTeles, A. 2011. Dietary carbohydrate utilization 


\section{SOUZA, MELO, NOGUEIRA FILHO, CAMPECHE E FIGUEIREDO}

by European sea bass (Dicentrarchus labrax L.) and gilthead sea bream (Sparus aurata L.) juveniles. Fish Sci, 19: 201-215.

Fabregat, T.E.H.P.; Pereira, T.S.; Boscolo, C.N.; Alvarado, J.D. e Fernandes, J.B.K. 2011. Substituição da farinha de peixe pelo farelo de soja em dietas para juvenis de curimba. Bol Inst Pesc, 37: 289-294.

Getachew, G.; Makkar, H.P.S. and Becker, K. 2000. Effect of polyethylene glycol on in vitro degradability of nitrogen and microbial protein synthesis from tannin-rich browse and herbaceous legumes. Brit J Nutrit, 48: 73-83.

Gonçalves, G.S.; Furuya, W.M.; Ribeiro, R.P.; Furuya, V.R.B. e Soares, C.M. 2002. Farelo de canola na alimentação do piavuçu, Leporinus macrocephalus (Garavello \& Britski), na fase inicial. Acta Sci, 24: 921-925.

Honorato, C.A.; Almeida, L.C.; Silva Nunes, C.; Carneiro, D.J. and Moraes, G. 2009. Effects of processing on physical characteristics of diets with distinct levels of carbohydrates and lipids: the outcomes on the growth of pacu (Piaractus mesopotamicus). Aquaculture, 16: 91-99.

Jesus, L.S.F.; Azevedo, R.V.; Carvalho, J.S.O. e Braga, L.G.T. 2011. Farelos da vagem da algaroba e da folha da mandioca em rações para juvenis de tilápia do Nilo mantidos em água salobra. Rev Bras Saúde Prod Anim, 12: 11161125.

Lemos, M.V.A.; Guimarães, I.G. e Miranda, E.C. 2011. Farelo de coco em dietas para o tambaqui (Colossoma macropomum). Rev Bras Saúde Prod Anim, 12: 188-198.

Lima, C.B.; Guimarães, I.G.; Ribeiro, V.L. e Miranda, E.C. 2009. Farinha de algaroba em dietas para tilápia do Nilo (Oreochromis niloticus). Pubvet, 3: 491-496.

Lima, M.R.; Ludke, M.C.M.M.; Porto-Neto, F.F.; Pinto, B.W.C.; Torres, T.R. e Souza, E.J.O. 2011. Farelo de resíduo de manga para tilápia do Nilo. Acta Scient, 33: 65-71.

Liti, D.M.; Mugo, R.M.; Munguti, J.M. and Waidbacher, H. 2006. Growth and economic performance of Nile tilapia (Oreochromis niloticus L.) fed on three brans (maize, wheat and rice) in fertilized ponds. Aquac Nutr, 12: 239-245.

Melo, J.F.B.; Seabra, A.G.L.; Souza, S.A.; Souza, R.C. e Figueiredo, R.A.C.R. 2012. Substituição do farelo de milho pela farinha de manga no desempenho da tilápia-do-Nilo. Arq Bras Med Vet Zoo, 64: 177-182.

Monteiro, B.A. 2009. Valor nutricional de partes convencionais e não convencionais de frutas e hortaliças. Dissertação (Mestrado em Energia na Agricultura). Universidade Estadual Paulista Júlio de Mesquita Filho. Faculdade de Ciências Agronômicas. Botucatu-SP. 62 pp.

Muñoz-Ramírez, A.P. 2005. Utilização de carboidratos digestíveis em dietas para pacu, Piaractus mesopotamicus (Holmberg, 1887). Tese (Doutorado em Aquicultura). Universidade Estadual Paulista. Centro de Aqüicultura. Jaboticabal-SP. 116 pp.

Oishi, C.A. 2007. Resíduo da castanha da Amazônia (Bertholletia excelsa) como ingrediente em rações para juvenis de tambaqui (Colossoma macropomum). Dissertação (Mestrado em Ciências Biológicas). Instituto Nacional de Pesquisas da Amazônia. Universidade Federal da Amazônia. Manaus-AM. 61 pp.

Oliveira Filho, P.R.C. e Fracalossi, D.M.F. 2006. Coeficientes de digestibilidade aparente de ingredientes para juvenis de jundiá. Rev Bras Zootecn, 35: 1581-1587.

Pedron, F.A.; Radünz Neto, J.; Silva, L.P.; Bergamin, G.T.; Maschio, D.; Martinelli, S.G.; DellaFlora, M.A. e Corrêia, V. 2011. Crescimento de juvenis de jundiá (Rhamdia quelen) com diferentes proporções de amilose: amilopectina na dieta. Arq Bras Med Vet Zoo, 63: 1200-1207.

Pereira-da-Silva, E.M. e Pezzato, L.E. 2000. Respostas da tilápia do Nilo (Oreochromis niloticus) à atratividade e palatabilidade de ingredientes utilizados na alimentação de peixes. Rev Bras Zootecn, 29: 1273-1280.

Perosa, J.M.Y.; Silva, C.S. e Arnaldi, C.R. 2009. Avaliação das perdas de manga (Mangifera indica L.) no mercado varejista da cidade de Botucatu. Rev Bras Frutic, 31: 732-738.

Pimenta, C.J.;Oliveira, M.M.; Ferreira, L.O.; Pimenta, M.E.S.G.; Logato, P.V.R.; Leal, R.S. e Murgas L.D.S. 2011. Aproveitamento do resíduo do café na alimentação de tilápia do nilo. Arch Zootec, 60: 583-593.

Ramos, A.P.S.; Carvalho, J.S.O.; Azevedo, R.V.; Sena, M.F.; Oliveira, D.A.; Rodrigues, F.L. e Braga, L.G.T. 2008. Utilização do farelo da folha da mandioca na alimentação de tilápias. In: V Congresso Nordestino de Produção Animal. 


\section{FARINHA DE MANGA E CRESCIMENTO E COMPOSIÇÃO CORPORAL DA TILÁPIA}

Anais... Aracaju. pp. 1-3.

Rawles, S. and Lochmann, R. 2003. Effects of amylopectin/amylose starch ratio on growth, body composition and glycemic response of sunshine bass (Morone chrysops $\times M$ saxatilis). J World Aquac Soc, 34: 278-288.

Rostagno, H.S.; Albino, L.F.T.; Donzele, J.L.; Gomes, P.C.; Oliveira, R.F.; Lopes, D.C.; Ferreira, A.S. e Barreto, S.L.T. 2005. Tabelas brasileiras para aves e suínos: composição de alimentos e exigências nutricionais. $2^{\underline{a}}$ ed. UFV. Viçosa. $141 \mathrm{pp}$.

Santos, E.L.; Ludke, M.C.M.M.; Barbosa, J.M.; Rabello, C.B.V.; Ludke, J.V.; Winterle, W.M.C.e Silva, E.G. 2009. Níveis de farelo de coco em rações para alevinos de tilápia do Nilo. Rev Bras Saúde Prod Anim, 10: 390-397.

Shahidi, F. and Naczk, M. 1995. Food phenolics: souces, chemistry, effects and applications. Technomic Publishing Co. Lancaster. pp. 235273.

Signor, A.A.; Boscolo, W.R.; Feiden, A.; Reidel, A.; Signor, A. e Grosso, I.R. 2007. Farinha de vísceras de aves na alimentação de alevinos de piavuçu (Leporinus macrocephalus). Ciênc
Rural, 37: 828-834.

Silva, F.A.S. 2010. ASSISTAT. Versão 7.5 beta. http://www.assistat.com. DEAG-CTRN-UFCG (08/02/2011)

Silva, D.J. e Queiroz, A.C. 2002. Análise de alimentos: métodos químicos e biológicos. $3^{\mathrm{a}}$ ed. Universidade Federal de Viçosa. Viçosa-MG. $235 \mathrm{pp}$.

Tachibana, L.; Gonçalves, G.S.; Guimarães, I.G.; Falcon, D.R.; Barros, M.M. e Pezzato, L.E. 2010. Substituição do milho pelo triticale na alimentação de tilápias-do-nilo. Rev Bras Zootecn, 39: 241246.

Teixeira, E.A.; Crepaldi, D.V.; Faria, P.M.C.; Ribeiro, L.P.; Melo, D.C.; Euler, A.C.C. e Saliba, E.O.S. 2006. Substituição de farinha de peixes em rações para peixes. Rev Bras Reprod Anim, 30: 118-125.

Vieira, P.A.F.; Queiroz, J.H.; Albino, L.F.T.; Moraes, G.H.K.; Barbosa, A.A.; Müller, E.S. e Viana, M.T.S. 2008. Efeitos da inclusão de farelo resíduo de manga no desempenho de frangos de corte de 1 a 42 dias. Rev Bras Zootecn, 37: 2173-2178. Wilson, R.P. 1994. Review: utilization of dietary carbohydrate by fish. Aquaculture, 124: 67-80. 